\title{
Cytoplasmic droplet acting as a mitochondrial modulator during sperm maturation in dogs
}

\author{
D.S.R. Angrimani ${ }^{\mathrm{a}}$, J.D.A. Losano ${ }^{\mathrm{a}}$, C.F. Lucio $^{\mathrm{a}}$, G.A.L. Veiga ${ }^{\mathrm{a}}$, F.C. Landim ${ }^{\mathrm{b}}$, \\ M. Nichi ${ }^{\mathrm{a}}$, C.I. Vannucchi ${ }^{\mathrm{a}, *}$ \\ a Department of Animal Reproduction, School of Veterinary Medicine and Animal Science, University of São Paulo, Av. Prof. Orlando Marques de \\ Paiva, 87, 05508-270, São Paulo, Brazil \\ b Department of Animal Reproduction, School of Veterinary Medicine and Animal Science, São Paulo State University - UNESP, Botucatu, São Paulo, \\ Brazil
}

\section{A R T I C L E I N F O}

\section{Keywords:}

Cytoplasmic droplet

Sperm maturation

Epididymis

Dogs

\begin{abstract}
A B S T R A C T
Motility acquisition during sperm maturation and passage through the epididymis is closely related to mitochondrial function and appears to occur in parallel with cytoplasmic droplet (CD) migration. However, such mechanism remains unclear in dogs. Thus, the aim of this study was to characterize the influence of sperm $C D$ in the mitochondrial functionality during epididymal sperm maturation in dogs. Twenty-one adult dogs were submitted to elective bilateral orchiectomy. Testicles were stored for $18-24 \mathrm{~h}$ at $5{ }^{\circ} \mathrm{C}$ and epididymal sperm samples were then collected from different segments of the epididymis (caput, corpus and cauda). Samples were evaluated for computer-assisted motility analysis (CASA), presence of CD (eosin/nigrosin stain), ultrastructural $\mathrm{CD}$ analysis and sperm mitochondrial activity (3,3' diaminobenzidine technique) and membrane potential (JC-1 probe). Samples collected from the corpus epididymis showed higher motility and mitochondrial activity in comparison to the caput sperm. Moreover, corpus sperm had lower percentage of proximal droplets compared to caput samples, while mitochondrial membrane potential remained unchanged. Cauda samples showed higher motility, mitochondrial activity and potential, however, lower presence of sperm droplets (proximal and distal). In conclusion, the $\mathrm{CD}$ is essential for epididymal sperm maturation in dogs, showing important functions along the transit in the epididymis. In the corpus segment, the migration of the $\mathrm{CD}$ along the sperm midpiece provides a high mitochondrial activity and the onset of sperm motility. On the other hand, sperm from cauda epididymis lack CD but suffered lipid membrane changes which allow a maximum mitochondrial membrane potential and motility.
\end{abstract}

\section{Introduction}

During spermatogenesis, germinative cell cytoplasm is phagocytozed by the Sertoli cells, and only a remnant residue persists, forming the sperm cytoplasmic droplet (CD) (Cooper, 2005). In the caput epididymis, high percentage of spermatozoa possess a proximal droplet, however in the epididymal sperm maturation course, the CD migrates from a proximal to a distal position on the midpiece (Cooper, 2011). Simultaneously to CD migration, canine spermatozoa acquire motility in the corpus epididymis (Varesi et al., 2013; Angrimani et al., 2014a).

The exact mechanism related to the CD migration and the motility gain remains uncertain (Xu et al., 2013). Yuan et al. (2013) in

\footnotetext{
* Corresponding author.

E-mail address: cacavann@usp.br (C.I. Vannucchi).
} 
mice, reported an energy source function of the CD during sperm maturation in the epididymis, suggesting that the droplet contains enzymes and substrates required for the proper functioning of sperm mitochondria. Moreover, several studies indicate that a failure during the translocation of the CD decrease sperm motility (Nothling et al., 1997; Cooper, 2005; Xu et al., 2013), which show that an important relationship between $\mathrm{CD}$, mitochondrial function and sperm motility exists.

The relation between $\mathrm{CD}$ and sperm motility can be attributed to several typical features of the CD, such as the unique composition of lipids, RNAs, lipoproteins and hydrolytic enzymes in the cytoplasm residue (Cooper and Yeung, 2003; Rengan et al., 2012). Moreover, the CD is constituted of elements with Golgi characteristics, which are incorporated to the spermatic cell during droplet migration along the sperm maturation, been related to plasma membrane modifications (Oko et al., 1993; Cortadellas and Durfort, 1994) and acquisition of sperm motility (Xu et al., 2013).

In this context, studies have focused on the function of the $\mathrm{CD}$ and the pathways involved in motility acquisition. Therefore, the inhibition of sperm motility can contribute to the physiological knowledge of sperm maturation and ultimately allow the development of new male contraceptive or the elaboration of treatments for male infertility related to the loss of sperm motility (Ma et al., 2013). Despite such state of art, there are still few studies using the canine species, which is considered the experimental model of choice for the man (Kirchhoff, 2002). Thus, the aim of this study was to verify the influence of the sperm CD in the mitochondrial functionality during epididymal sperm maturation in dogs.

\section{Materials and Methods}

\subsection{Animals}

The present study was approved by the Bioethics Committee of the School of Veterinary Medicine and Animal Science-University of São Paulo (protocol number: 2277/2011).

Twenty one healthy mature dogs, aged from 1 to 6 years, of different breeds (Poodle, Mixed Breed, Labrador Retriever, German Shepherd, Shetland Sheepdog and Schnauzer) and body weights $(17.41 \pm 2.6 \mathrm{~kg})$ were used. All animals were privately owned and fed a commercial diet for dogs; water was provided ad libitum. Furthermore, animals were tested against brucellosis, using the Antigen Rapid Canine Brucella Ab Ttest (Bioeasy, Minas Gerais, Brazil), detecting the anti-Brucella canis IgG antibodies.

\subsection{Sample collection}

Animals were submitted to bilateral orchiectomy, and testicles-epididymis were immediately stored at $5{ }^{\circ} \mathrm{C}$ for not less than $18 \mathrm{~h}$ and not more than $24 \mathrm{~h}$. Epididymal sperm samples were collected by performing incisions in the caput, corpus and cauda epididymides (Angrimani et al., 2017). Dissections were performed carefully to avoid cutting blood vessels and several incisions were performed in each epididymal region (caput, corpus and cauda) which were individualized using a hemostatic forceps. The flowing epididymal fluid was collected with an automatic pipette, and the total volume of the epididymal sample was resuspended in $300 \mu \mathrm{L}$ of phosphate buffered saline medium (PBS). The dilution rate varied according to the epididymal size, similar to a previously described protocol (Kaabi et al., 2003). Tubes containing solely the PBS medium were weighted before (tube + PBS) and after the addition of the epididymal sample (tube + PBS + epididymal fluid) to calculate the epididymal fluid dilution factor. Due to the small volume recovered, the samples from the left and right epididymides of the same animal were pooled.

\subsection{Assessment of sperm motility}

Samples from the epididymal segments (i.e. caput, corpus and cauda) were immediately evaluated for computer-assisted sperm analysis (CASA; HTM-IVOS Ultimate 12.3; Hamilton Thorne Biosciences, Beverly, MA, USA), according to a previously described protocol (Iguer-ouada and Verstegen, 2001). Briefly, $10 \mu \mathrm{L}$ of each sample was deposited on microscope slides previously warmed at $37^{\circ} \mathrm{C}$ and covered by coverslips. Eight fields of view were randomly selected and the following variables were assessed: VAP (average pathway velocity, $\mu \mathrm{m} / \mathrm{s}$ ), VSL (strait-line velocity, $\mu \mathrm{m} / \mathrm{s}$ ), VCL (curvilinear velocity, $\mu \mathrm{m} / \mathrm{s}$ ), ALH (amplitude of lateral head displacement, $\mu \mathrm{m}$ ), BCF (beat cross frequency, Hz), STR (straightness-VSL/VAP, \%), LIN (Linearity-VSL/VCL,\%); MOT (motility, $\%$ ), PROG (progressive motility, \%). Sperm were also divided into four groups based on velocity: rapid (RAP, VAP > $50 \mu \mathrm{m} / \mathrm{s}, \%$ ), medium (MED, $30 \mu \mathrm{m} / \mathrm{s}<\mathrm{VAP}<50 \mu \mathrm{m} / \mathrm{s}$, \%), slow (SLOW, VAP $<30 \mu \mathrm{m} / \mathrm{s}$ or VSL $<15 \mu \mathrm{m} / \mathrm{s}$, \%) and non-moving spermatozoa (STATIC, \%).

\subsection{Evaluation of mitochondrial activity and presence of cytoplasmic droplets}

To assess mitochondrial activity we used the cytochemical technique of 3,3' diaminobenzidine solution (1 mg/mL of DAB in PBS), which categorize the sperm into four classes: high (DAB-Class I), medium (DAB-Class II), low (DAB-Class III) and absence (DAB-Class IV) of mitochondrial activity (Hrudka, 1987). For this purpose, a sperm sample was incubated under light at $37{ }^{\circ} \mathrm{C}$ for $1 \mathrm{~h}$ with DAB in a ratio of $1: 1(20 \mu \mathrm{L}$ of sample in $20 \mu \mathrm{L}$ of DAB). After this period, samples were smeared on glass slides with subsequent fixation in $10 \%$ of formalin for $15 \mathrm{~min}$. The evaluation was performed with a light transmitted microscope under $1000 \times$ magnification (Nikon, Eclipse E200, Japan) by counting 200 cells. The results were expressed in percentage (\%).

To evaluate the presence of CDs, Eosin/Nigrosin stain was used. In brief, $5 \mu \mathrm{L}$ of semen and $5 \mu \mathrm{L}$ of the previously prepared stain were placed in a pre-warmed glass slide. The sperm smear was evaluated under light microscopy (Nikon, Eclipse E200, Japan) at 
$1000 \times$ magnification. Sperm CD was classified as proximal and distal considering the presence and position of the droplet in the sperm midpiece (Barth and Oko, 1989).

\subsection{Assessment of mitochondrial membrane potential}

Flow cytometry was performed with the use of the Guava EasyCyte Mini System (Guava Technologies, Hayward, CA, USA) with fluorescent probe to specifically analyze the mitochondrial membrane potential (MMP), with a $488 \mathrm{~nm}$ laser argon and the following filters (photodetector): PM1 $(680 \mathrm{~nm})$ to red and PM3 $(525 \mathrm{~nm})$ to green. For data analysis, cell doublets and debris were excluded using PM3/FSC (forward scatter). A minimum of 20,000 spermatozoa were examined for each assay. The MMP was determined with the use of 5, 5',6, 6', tetrachloro-1, 1', 3, 3'-tetraethyl-benzimidazolcarbocyanine iodide (JC-1; Sigma Aldrich Chemical Co., Germany) (Lucio et al., 2016). For this purpose, $2 \mu \mathrm{L}$ of JC1 probe ( $1 \mu \mathrm{M}$ in dimethyl sulfoxide) was added to 7 million spermatozoa. After $8 \mathrm{~min}$ at $37{ }^{\circ} \mathrm{C}, 300 \mu \mathrm{L}$ of PBS was added and analyzed by flow cytometry. The sperm population was selected by red fluorescence and then evaluated in the dot plot for yellow fluorescence. Sperm were separated into two populations: high MMP and low MMP. For spermatozoa with high mitochondrial potential, JC-1 formed complexes known as J-aggregates, with intense red fluorescence. For sperm with low mitochondrial membrane potential, JC-1 remained in the monomer form, which appears only as green fluorescence. Data were analyzed using FlowJo v8.7 Software (Flow Cytometry Analysis Software-Tree Star Inc., Ashland, OR, USA).

\subsection{Ultrastructural analysis}

Samples were processed following the protocol described by Long et al. (1996), for which the sperm pellet was initially fixed with $2.5 \%$ glutaraldehyde in $0.1 \mathrm{M}$ phosphate buffer ( $\mathrm{pH} 7.4$ ) and then in $1 \%$ osmium tetroxide in the same buffer. Samples dehydration took place in a series of increasing acetone concentration. Then, dehydrated sperm were included in plastic resin (Embed 812, Electron Microscopy Sciences). Ultrathin sections succeeded with a diamond knife (70-90 nm of thickness) and were mounted on copper grids and stained with uranyl acetate and lead citrate (Sato et al., 2008). The samples were examined in a transmission electron microscope (TEM-Philips EM 301, USA). Fifteen images were randomly chosen from each epididymal segment (caput, corpus and cauda) and described according to the morphological evaluation suggested by Barth and Oko (1989).

\subsection{Statistical analysis}

All data were analyzed using the SAS for Windows (SAS Institute Inc., Cary, NC, USA, 2000). The effect of the sperm origin (epididymal spermatozoa from caput, corpus and cauda) was determined using parametric (Tukey test) and nonparametric (Wilcoxon) tests, according to the residue normality (Gaussian distribution) and variance homogeneity of each variable. A probability value of $\mathrm{P}<0.05$ was considered statistically significant. The results are reported as untransformed means \pm SEM. Pearson and Spearman (parametric and no parametric variables, respectively) correlations were used to calculate the relationship between the variables studied in each variable group.

In order to perform the ultrastructural analysis, ten animals were selected through randomization PROC PLAN (seed: 3859012) SAS System for Windows program (SAS Institute Inc., Cary, NC, USA, 2000). These animals were placed in the three experimental groups, according to the epididymal segment (caput, corpus, cauda). The results of the ultrastructural analysis were descriptive.

\section{Results}

The Computer Assisted Semen Analysis (CASA) showed significant and progressive increase in sperm kinetics during the sperm epididymal transit, represented by motility, progressive motility, rapid speed, average path velocity (VAP), straight line velocity (VSL), curvilinear velocity (VCL), straightness (STR) and linearity (LIN) (Table 1). The percentage of static spermatozoa from the epididymis caput was greater than corpus and cauda sperm (Table 1). However, the caput sperm showed lower results of medium speed, beat cross frequency (BCF) and amplitude of lateral head displacement (ALH) when compared with corpus and cauda sperm (Table 1).

In relation to the analysis of mitochondrial membrane potential, the samples from cauda epididymides showed higher values $(65 \pm 4.2 \%)$ compared to corpus $(38.2 \pm 3.6 \%)$ and caput sperm (43.6 $\pm 4.1 \%)$ (Fig. 1). Moreover, considering the high mitochondrial activity analysis (DAB-Class I), cauda sperm presented higher percentage (75.9 $\pm 3.4 \%$ ) compared to the corpus $(46.8 \pm 2.5 \%)$ and caput sperm $(26 \pm 2.4 \%)$ (Fig. 2). On the other hand, the absence of mitochondrial activity (DAB-Class IV) was lower in cauda sperm $(12.5 \pm 3 \%)$, in comparison to corpus $(26.2 \pm 3 \%)$ and caput sperm (39.5 $\pm 3.6 \%-$ Fig. 2$)$. The medium (DAB-Class II) and low (DAB-Class III) mitochondrial activity in the caput (21.6 $\pm 1.9 \%$ and $13.2 \pm 1.6 \%$, respectively) and corpus $(17.4 \pm 1.7 \%$ and $9.3 \pm 1.2 \%$, respectively) were different from cauda sperm $(9 \pm 1.1 \%$ and $2.9 \pm 0.6 \%$, respectively-Fig. 2).

Regarding the presence of CDs assessed by the eosin/nigrosine technique, we observed a higher percentage of proximal droplet in the caput segment $(71 \pm 2.3 \%)$, followed by corpus $(20.1 \pm 5 \%)$ and cauda sperm $(10.3 \pm 5 \%$-Fig. 3$)$. On the other hand, cauda $(6.6 \pm 1.3 \%)$ and corpus sperm $(6.4 \pm 1 \%)$ presented higher percentage of distal droplet compared to caput sperm (0.7 $\pm 0.4 \%$-Fig. 3).

The ultrastructural analysis revealed a great number of normal spermatozoa in the epididymis cauda (Fig. 4), without acrosomal 
Table 1

Effect of sperm origin (cauda, corpus and caput epididymis) on computer assisted sperm analysis (CASA) in dogs.

\begin{tabular}{|c|c|c|c|}
\hline & Caput & Corpus & Cauda \\
\hline$\%$ of Motile sperm & $0.0 \pm 0.0^{\mathrm{C}}$ & $34.5 \pm 3.5^{\mathrm{B}}$ & $71.0 \pm 4.4^{\mathrm{A}}$ \\
\hline$\%$ of Progressive sperm & $0.0 \pm 0.0^{\mathrm{B}}$ & $5.5 \pm 0.9^{\mathrm{B}}$ & $27.8 \pm 2.9^{\mathrm{A}}$ \\
\hline$\%$ of Rapid sperm & $0.0 \pm 0.0^{\mathrm{C}}$ & $18.2 \pm 2.4^{\mathrm{B}}$ & $54.2 \pm 4.5^{\mathrm{A}}$ \\
\hline$\%$ of Medium velocity sperm & $0.0 \pm 0.0^{\mathrm{B}}$ & $16.3 \pm 1.7^{\mathrm{A}}$ & $16.8 \pm 2.6^{\mathrm{A}}$ \\
\hline$\%$ of Slow sperm & $6.0 \pm 2.5$ & $6.0 \pm 2.4$ & $10.5 \pm 1.4$ \\
\hline$\%$ of Static sperm & $94.0 \pm 2.5^{\mathrm{A}}$ & $54.8 \pm 4.4^{\mathrm{B}}$ & $23.1 \pm 3.6^{\mathrm{C}}$ \\
\hline Average path velocity (VAP; $\mu \mathrm{m} / \mathrm{s}$ ) & $0.0 \pm 0.0^{\mathrm{C}}$ & $66.3 \pm 5.5^{\mathrm{B}}$ & $94.9 \pm 5.6^{\mathrm{A}}$ \\
\hline Straight line velocity (VSL; $\mu \mathrm{m} / \mathrm{s}$ ) & $0.0 \pm 0.0^{\mathrm{C}}$ & $37.8 \pm 3.7^{\mathrm{B}}$ & $67.2 \pm 4.6^{\mathrm{A}}$ \\
\hline Curvilinear velocity (VCL; $\mu \mathrm{m} / \mathrm{s}$ ) & $0.0 \pm 0.0^{\mathrm{C}}$ & $142.5 \pm 10.3^{\mathrm{B}}$ & $178.5 \pm 10.0^{\mathrm{A}}$ \\
\hline Amplitude of lateral head displacement (ALH; $\mu \mathrm{m})$ & $0.0 \pm 0.0^{B}$ & $7.3 \pm 0.6^{\mathrm{A}}$ & $7.8 \pm 0.3^{\mathrm{A}}$ \\
\hline Beat cross frequency (BCF; Hz) & $0.0 \pm 0.0^{\mathrm{B}}$ & $32.0 \pm 3.1^{\mathrm{A}}$ & $28.3 \pm 2.2^{\mathrm{A}}$ \\
\hline Straightness (STR; \%) & $0.0 \pm 0.0^{\mathrm{C}}$ & $48.9 \pm 3.5^{\mathrm{B}}$ & $67.4 \pm 1.4^{\mathrm{A}}$ \\
\hline Linearity (LIN; \%) & $0.0 \pm 0.0^{\mathrm{C}}$ & $26.3 \pm 2.4^{B}$ & $38.9 \pm 1.5^{\mathrm{A}}$ \\
\hline
\end{tabular}

Different superscripts in the same line indicate significant differences $(\mathrm{P}<0.05)$.

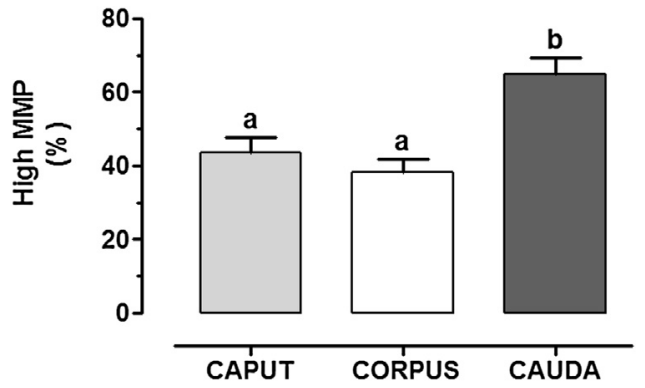

Fig. 1. Mean and standard error $(\mathrm{X} \pm \mathrm{SE}$ ) of the high mitochondrial membrane potential (MMP_\%) analyzed by flow cytometry (JC-1 probe) in epididymal sperm according to the original segment (caput, corpus and cauda) in dogs.Different superscripts indicate significant differences between epididymal segments ( $<$ $<.05$ ).

defects and with a less frequency of CDs. However, samples from the epididymis caput (Fig. 5) had abnormalities in the midpiece, such as proximal CD consisting of a system of vesicles and vacuoles surrounded by the plasma membrane. It is possible to observe the axoneme and mitochondrial sheath in the center of the CD. Samples from cauda and corpus also presented distal CD, located behind the midpiece (Fig. 6).

Considering the correlation analysis, there was a positive correlation $(r=0.53$ and $p=0.01)$ between the percentage of absent mitochondrial activity (DAB-Class IV) and static spermatozoa in the caput epididymis. In the cauda segment we observed a positive correlation between the percentage of high mitochondrial activity (DAB-Class I) and sperm motility $(\mathrm{r}=0.45$ and $\mathrm{p}=0.05$ ), progressive sperm $(r=0.44$ and $p=0.05)$ and rapid speed $(r=0.51$ and $p=0.02)$.

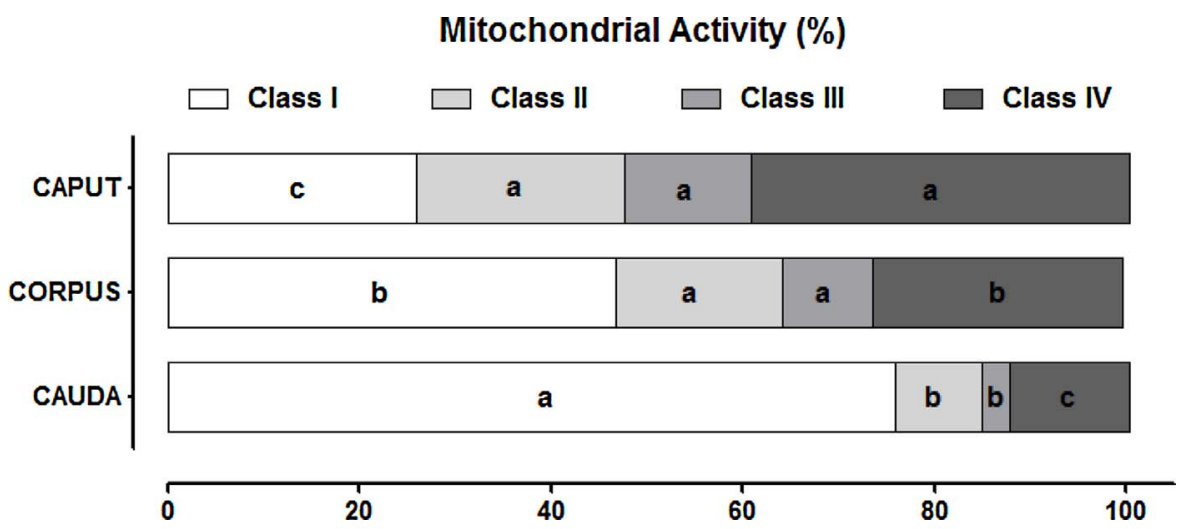

Fig. 2. Mean and standard error $(X \pm S E)$ of the mitochondrial activity (\%) analyzed by the cytochemical technique of 3,3' diaminobenzidine (DAB) in epididymal sperm according to the original segment (caput, corpus and cauda) in dogs.Different superscripts in the same mitochondrial activity class indicate significant differences between epididymal origin ( $\mathrm{P}<0.05$ ). DAB —Class I: high; DAB-Class II: medium; DAB-Class III: low and DAB-Class IV: absence of mitochondrial activity. 


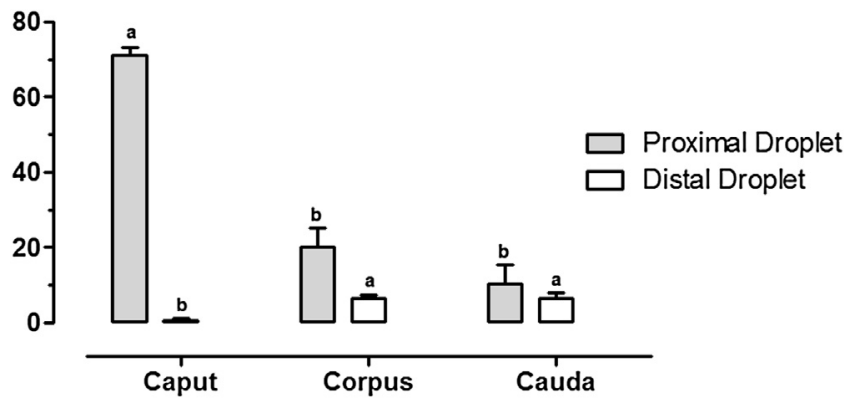

Fig. 3. Mean and standard error $(\mathrm{X} \pm \mathrm{SE}$ ) of the percentage of proximal and distal droplets (\%) in epididymal sperm according to the original segment (caput, corpus and cauda) in dogs.Different superscripts indicate significant differences $(\mathrm{P}<0.05)$ between epididymal segments.

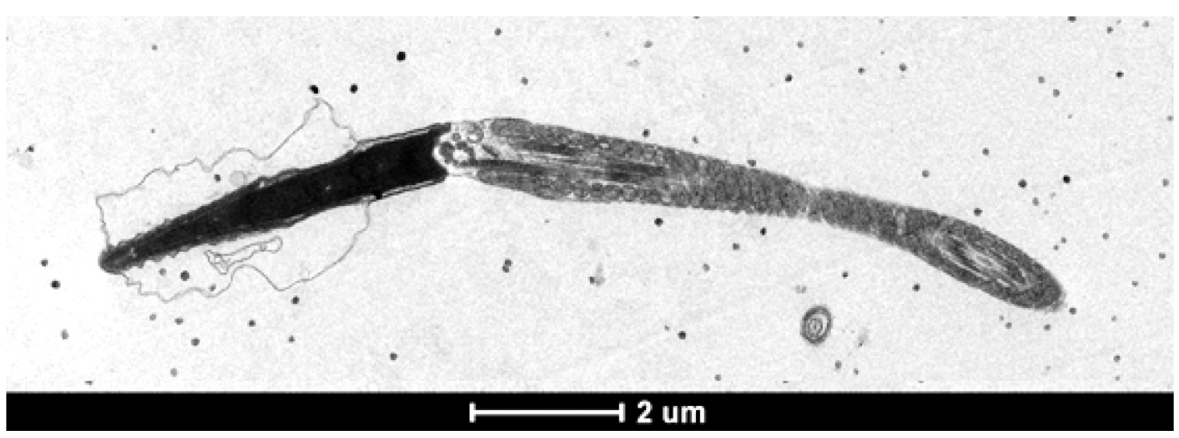

Fig. 4. Transmission electron microscopy of cauda epididymis region showing a sagittal section of a normal dog spermatozoa.

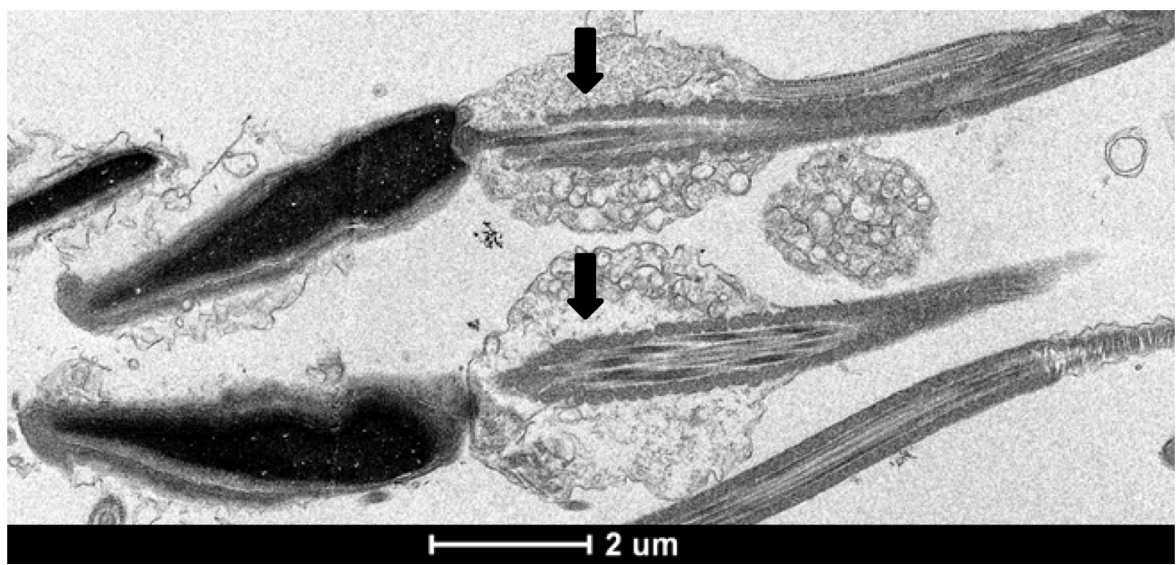

Fig. 5. Transmission electron microscopy of caput epididymis region with a sagittal section of a dog spermatozoa with a proximal CD (arrows).

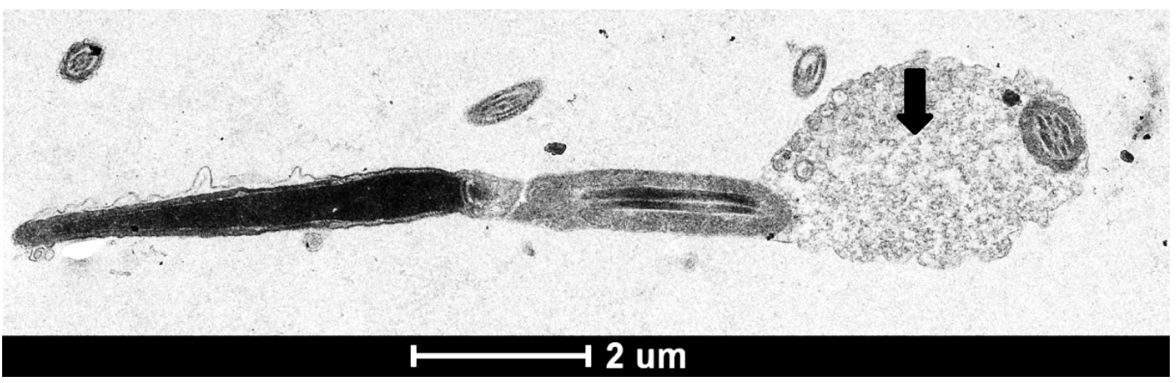

Fig. 6. Transmission electron microscopy of corpus epididymis region showing a sagittal section of a dog spermatozoon with a distal CD (arrow). 


\section{Discussion}

In the present study, we evaluated the influence of the CD on sperm motility and mitochondrial functionality (potential and activity) during the epididymal sperm maturation in dogs.

The CD migrates along the spermatozoa from the proximal to the distal region of the midpiece during sperm maturation (Cooper, 2011). In dogs, this translocation occurs in corpus epididymis segment (Varesi et al., 2013; Angrimani et al., 2014a). Moreover, we demonstrated previously that spermatozoa initiate the process of motility in the epididymis corpus (Angrimani et al., 2014a, 2014b). In this study, we additionally show that the CD migration occurs also in the epididymis corpus. In fact, there was a decrease in the percentage of proximal droplets between the caput and corpus of the epididymis considering the eosin/nigrosine stain and confirmed by the ultrastructural analysis. Thus, it is possible to establish a probable relationship between the migration of the CD and the acquisition of sperm motility during the transit from the caput to the corpus epididymis in dogs.

The exact mechanism by which the CD influences sperm motility remains not elucidated (Xu et al., 2013). The CD is considered a remnant residue of germ cell cytoplasm (Cooper, 2005), composed by lysosomal hydrolases and metabolic enzymes (e.g. alkaline phosphatase, lactic dehydrogenase and transaldolase), involved in energy metabolism (Bavdek and Glover, 1970; Garbers et al., 1970; Moniem and Glover, 1972; Oko et al., 1993). Moreover, Yuan et al. (2013) reported that the CD can act as a source of substances (e.g. enzymes and substrates) used mainly by the glycolytic pathway. Therefore, pyruvate (final product of the glycolytic pathway) is considered to participate in mitochondrial activation. Thus, we can hypothesize that during the migration of the CD in the sperm midpiece, its components are incorporated by sperm mitochondria, enabling the production of sperm energy in dogs. In fact, in this study we observed the CD migration from the proximal to distal region of the midpiece concomitant with a gradual acquisition of mitochondrial activity (assessed by DAB technique) during the sperm maturation. Moreover, the epididymis caput presented a greater number of spermatozoa without mitochondrial activity (DAB-Class IV), while in corpus epididymis there was an increased percentage of sperm with high mitochondrial activity (DAB-Class I), indicating a gain of mitochondrial activity from caput to the corpus epididymis. The evaluation of the mitochondrial activity aims to observe the electron transfer efficiency between the mitochondrial enzyme complexes (Terada, 1990), which is essential for ATP production through oxidative phosphorylation and to provide sperm motility acquisition (Fariello et al., 2012). The positive correlation between the absence of mitochondrial activity (DAB-Class IV) and static spermatozoa in the caput epididymis in this study clearly demonstrates a relationship between the absence of mitochondrial activity and sperm immobility. Thus, it is possible to infer that the CD influences the onset of mitochondrial activity of epididymal spermatozoa, initiating early epididymal sperm motility in the corpus segment.

On the other hand, no difference in the mitochondrial membrane potential (MMP) between caput and corpus sperm was observed. It is important to point out that mitochondrial activity and mitochondrial membrane potential are two separate processes. The mitochondrial membrane potential is related to the ability of the mitochondria to pump protons from the mitochondrial matrix to the intermembrane space in order to produce an electrochemical gradient, utilizing the free energy generated by electron transport (Chen, 1988; Saraste, 1999). Studies have shown that the mitochondria has low membrane potential during uncoupled oxidative phosphorylation, but keeps its activity through electron transfer between the enzyme complex (Terada, 1990). In this context, it is possible to affirm that the sperm located in the corpus epididymis have high mitochondrial activity (DAB-Class I), however does not show maximum mitochondrial membrane potential. Previous studies in hamsters, monkeys and boars reported similar results, with low percentage of high MMP in the caput and corpus segments in relation to the epididymis cauda (Weissenberg et al., 1994; Aitken et al., 2007; Fabrega et al., 2012; Yuan et al., 2013). Therefore, we can hypothesize that the CD directly influences the onset of mitochondrial activity in the corpus epididymis, however the highest MMP, as well as the maximum sperm motility, will occur only in the cauda epididymis in dogs, where most of the droplets have already been removed (Angrimani et al., 2014a).

In our ultrastructural analysis, it was possible to observe a relationship between the epididymal sperm structure and the CD, showing that the droplets are adhered to the sperm and being surrounded by the lipid membrane of the spermatozoa. Thus, it is evident that during the $\mathrm{CD}$ migration along the spermatic flagellum a reorganization of the lipid membrane of the spermatozoon occurs (Schlegel et al., 1986; Bian et al., 2013). The changes in the lipid structure of the sperm plasma membrane, aiming an efficient transduction of ATP from the mitochondria, are essential for sperm motility (Amaral et al., 2013). Therefore, it is possible to suggest that the sperm from the epididymis cauda may have a better mitochondrial and flagellar functionality, since the CD migration has already been completed (Parks and Hammerstedt, 1985). In fact, we observed a positive correlation between high mitochondrial activity (DAB-Class I) and sperm motility, progressive and rapid speed in the cauda epididymis. Moreover, the higher motility kinetics, mitochondrial membrane potential and activity show a more efficient energetic distribution in the epidydimal cauda sperm, which has already passed through all morphological and functional changes offered by the CD migration along the epididymis.

\section{Conclusions}

In conclusion, the CD appears to be essential for sperm epididymal maturation in dogs, showing important functions along the transit in the epididymis. In the corpus segment, the migration of the $\mathrm{CD}$ along the sperm midpiece may provide a high mitochondrial activity and the onset of sperm motility. On the other hand, cauda epididymis sperm lack CD but suffered lipid membrane changes which can allow a maximum mitochondrial membrane potential and motility. Therefore, our results indicate that the $\mathrm{CD}$ translocation can provide a positive effect during the epididymal sperm transit, being essential for the sperm to endure the challenges of the post-ejaculatory environment. 


\section{Acknowledgements}

\section{The authors thank Dr. Camilla M Mendes and Prof. Mayra E O Assumpção for the flow cytometry analysis.}

\section{References}

Aitken, R.J., Nixon, B., Lin, M., Koppers, A.J., Lee, Y.H., Baker, M.A., 2007. Proteomic changes in mammalian spermatozoa during epididymal maturation. Asian J. Androl. 9, 554-564.

Amaral, A., Lourenco, B., Marques, M., Ramalho-Santos, J., 2013. Mitochondria functionality and sperm quality. Reproduction 146, R163-174.

Angrimani, D.S., Losano, J.D., Lucio, C.F., Veiga, G.A., Pereda, M.C., Nichi, M., Vannucchi, C.I., 2014a. Role of residual cytoplasm on oxidative status during sperm maturation in dogs. Anim. Reprod. Sci. 151, 256-261.

Angrimani, D.S., Lucio, C.F., Veiga, G.A., Silva, L.C., Regazzi, F.M., Nichi, M., Vannucchi, C.I., 2014b. Sperm maturation in dogs: sperm profile and enzymatic antioxidant status in ejaculated and epididymal spermatozoa. Andrologia 46, 814-819.

Angrimani, D.S.R., Nichi, M., Losano, J.D.A., Lucio, C.F., Veiga, G.A.L., Franco, M.V.J., Vannucchi, C.I., 2017. Fatty acid content in epididymal fluid and spermatozoa during sperm maturation in dogs. J. Anim. Sci. Biotechnol. 8, 18.

Barth, A.D., Oko, R.J., 1989. Abnormal Morphology of Bovine Spermatozoa. Iowa State University Press, Ames.

Bavdek, S., Glover, T.D., 1970. Alkaline phosphatase in the cytoplasmic droplet of rabbit spermatozoa. J. Reprod. Fertil. 22, 371-373.

Bian, X., Gandahi, J.A., Liu, Y., Yang, P., Zhang, L., Zhang, Q., Chen, Q., 2013. The ultrastructural characteristics of the spermatozoa stored in the cauda epididymidis in Chinese soft-shelled turtle Pelodiscus sinensis during the breeding season. Micron 44, 202-209.

Chen, L.B., 1988. Mitochondrial membrane potential in living cells. Annu. Rev. Cell Biol. 4, 155-181.

Cooper, T.G., 2005. Cytoplasmic droplets: the good, the bad or just confusing? Hum. Reprod. 20, 9-11.

Cooper, T.G., 2011. The epididymis, cytoplasmic droplets and male fertility. Asian J. Androl. 13, $130-138$.

Cooper, T.G., Yeung, C.H., 2003. Acquisition of volume regulatory response of sperm upon maturation in the epididymis and the role of the cytoplasmic droplet. Microsc. Res. Tech. 61, 28-38.

Cortadellas, N., Durfort, M., 1994. Fate and composition of cytoplasmic droplet of hamster epididymal spermatozoa. J. Morphol. 221, 199-210.

Fabrega, A., Puigmule, M., Bonet, S., Pinart, E., 2012. Epididymal maturation and ejaculation are key events for further in vitro capacitation of boar spermatozoa. Theriogenology 78, 867-877.

Fariello, R.M., Pariz, J.R., Spaine, D.M., Cedenho, A.P., Bertolla, R.P., Fraietta, R., 2012. Association between obesity and alteration of sperm DNA integrity and mitochondrial activity. BJU Intl. 110, 863-867.

Garbers, D.L., Wakabayashi, T., Reed, P.W., 1970. Enzyme profile of the cytoplasmic droplet from bovine epididymal spermatozoa. Biol. Reprod. 3, 327-337.

Hrudka, F., 1987. Cytochemical and ultracytochemical demonstration of cytochrome c oxidase in spermatozoa and dynamics of its changes accompanying ageing or induced by stress. Intl. J. Androl. 10, 809-828.

Iguer-ouada, M., Verstegen, J.P., 2001. Evaluation of the Hamilton Thorn computer-based automated system for dog semen analysis. Theriogenology 55, 733-749.

Kaabi, M., Paz, P., Alvarez, M., Anel, E., Boixo, J.C., Rouissi, H., Herraez, P., Anel, L., 2003. Effect of epididymis handling conditions on the quality of ram spermatozoa recovered post-mortem. Theriogenology 60, 1249-1259.

Kirchhoff, C., 2002. The dog as a model to study human epididymal function at a molecular level. Mol. Hum. Reprod. 8, 695-701.

Long, J.A., Wildt, D.E., Wolfe, B.A., Critser, J.K., DeRossi, R.V., Howard, J., 1996. Sperm capacitation and the acrosome reaction are compromised in teratospermic domestic cats. Biol. Reprod. 54, 638-646.

Lucio, C.F., Regazzi, F.M., Silva, L.C., Angrimani, D.S., Nichi, M., Vannucchi, C.I., 2016. Oxidative stress at different stages of two-step semen cryopreservation procedures in dogs. Theriogenology 85, 1568-1575.

Ma, L., Yu, H., Ni, Z., Hu, S., Ma, W., Chu, C., Liu, Q., Zhang, Y., 2013. Spink13, an epididymis-specific gene of the Kazal-type serine protease inhibitor (SPINK) family, is essential for the acrosomal integrity and male fertility. J. Biol. Chem. 288, 10154-10165.

Moniem, K.A., Glover, T.D., 1972. Alkaline phosphatase in the cytoplasmic droplet of mammalian spermatozoa. J. Reprod. Fertil. 29, 65-69.

Nothling, J.O., Gerstenberg, C., Volkmann, D.H., 1997. Semen quality after thawing: correlation with fertility and fresh semen quality in dogs. J. Reprod. Fertil. 51 (Supplement), 109-116.

Oko, R., Hermo, L., Chan, P.T., Fazel, A., Bergeron, J.J., 1993. The cytoplasmic droplet of rat epididymal spermatozoa contains saccular elements with Golgi characteristics. J. Cell Biol. 123, 809-821.

Parks, J.E., Hammerstedt, R.H., 1985. Development changes occurring in the lipids of ram epididymal spermatozoa plasma membrane. Biol. Reprod. 32, 653-668.

Rengan, A.K., Agarwal, A., van der Linde, M., du Plessis, S.S., 2012. An investigation of excess residual cytoplasm in human spermatozoa and its distinction from the cytoplasmic droplet. Reprod. Biol Endocrin. 10.

Saraste, M., 1999. Oxidative phosphorylation at the fin de siecle. Science 283, 1488-1493.

Sato, S., Adachi, A., Sasaki, Y., Ghazizadeh, M., 2008. Oolong tea extract as a substitute for uranyl acetate in staining of ultrathin sections. J. Microsc.-Oxford 229, $17-20$.

Schlegel, R.A., Hammerstedt, R., Cofer, G.P., Kozarsky, K., Freidus, D., Williamson, P., 1986. Changes in the organization of the lipid bilayer of the plasma membrane during spermatogenesis and epididymal maturation. Biol. Reprod. 34, 379-391.

Terada, H., 1990. Uncouplers of oxidative phosphorylation. Environ. Health Perspect. 87, 213-218.

Varesi, S., Vernocchi, V., Faustini, M., Luvoni, G.C., 2013. Morphological and acrosomal changes of canine spermatozoa during epididymal transit. Acta Vet. Scand. $55,17$.

Weissenberg, R., Yossefi, S., Oschry, Y., Madgar, I., Lewin, L.M., 1994. Investigation of epididymal sperm maturation in the golden hamster. Intl. J. Androl. 17, $256-261$.

Xu, H., Yuan, S.Q., Zheng, Z.H., Yan, W., 2013. The cytoplasmic droplet may be indicative of sperm motility and normal spermiogenesis. Asian J. Androl. 15, 799-805.

Yuan, S., Zheng, H., Zheng, Z., Yan, W., 2013. Proteomic analyses reveal a role of cytoplasmic droplets as an energy source during epididymal sperm maturation. PLoS ONE 8, e77466. 